\title{
A Case of Middle Ear Adenoma
}

\author{
Myung Joo Shim, Chan-II Song and Tae Hyun Yoon \\ Department of Otolaryngology, Asan Medical Center, University of Ulsan College of Medicine, Seoul, Korea
}

Received April 11, 2011

Revised January 5,2012

Accepted January 7, 2012

\author{
Address for correspondence \\ Tae Hyun Yoon, MD \\ Department of Otolaryngology, \\ Asan Medical Center, \\ University of Ulsan \\ College of Medicine, \\ 88 Olympic-ro 43-gil, Songpa-gu, \\ Seoul 138-736, Korea \\ Tel $+82-2-3010-3713$ \\ Fax $+82-2-489-2773$ \\ E-mail thyoon@amc.seoul.kr
}

\begin{abstract}
Middle ear adenoma can be a rare cause of a middle ear mass. These benign tumors can have epithelial and/or neuroendocrine differentiation. In 1976, Hyams was the first to describe a series of these tumors. It is still thought that these tumors are indistinguishable in composition from each other. We report a case of middle ear adenoma, the clinical and pathologic findings of which illustrate the biological behavior of the middle ear. A 17-year-old male presented with a history of recurrent, bloody otorrhea and high-pitched tinnitus. We diagnosed him with congenital cholesteatoma and performed surgery to remove the entire tumor as well as the incus. However, on postoperative pathology examination, we finally diagnosed the tumor as middleear adenoma with neuroendocrine differentiation. In the two years following surgery, the patient's adenoma has not recurred, although long-term observation will be required.
\end{abstract}

Korean J Audiol 2012;16:27-30

KEY WORDS: Middle ear · Adenoma · Ear neoplasm.

\section{Introduction}

Middle ear adenoma, a very rare type of middle ear tumor, was first reported by Hyams and Michaels ${ }^{1)}$ in 1976. Murphy, et al., ${ }^{2)}$ renamed it as carcinoid tumor which is similar to adenoma but is composed of neuroendocrine cells, and as seen on microscopic findings in 1980. Since then it has been called middle ear carcinoid tumor or middle ear adenoma.

In Korea, a total of three cases of adenomatous tumor in the middle ear have been reported. In 1978, Jun ${ }^{3)}$ reported a case of adenomatous carcinoma, and in 1991 and 2000, Kwon, et al. ${ }^{4)}$ and Jun, et al. ${ }^{5)}$ reported respective cases of adenomatous tumor, but no subsequently reported case has been found through a literature review.

As middle ear tumors with glandular structure can differentiate to neuroendocrine cells and epithelial cells, middle ear carcinoid tumor and adenoma were initially classified as different tumors, although they are currently considered by the majority of researchers to be the same low-grade middle ear tumor. ${ }^{2)}$ There is no clear definition regarding the derivation of the two tumors, although middle ear adenoma is divided into middle ear carcinoid tumor and middle ear adenoma. ${ }^{6}$

We report a case of a 17-year old male patient who presented with repeated otorrhea and tinnitus in the left ear.

The patient underwent surgical treatment for middle ear polyps and was diagnosed with middle ear adenoma based on the postoperative pathology report.

\section{Case Report}

A 17-year old male presented with a two-year history of tinnitus and intermittent bloody otorrhea in the left ear. He had no definitive abnormal findings based on his past medical history. He had no accompanying symptoms such as otalgia, dizziness or facial paralysis.

The right tympanic membrane was normal. The left tympanic membrane was not perforated but was observed as having a milky-white, middle ear polyp which has approximately $3 / 4$ of the eardrum protruded (Fig. 1).

Pure-tone audiometry showed normal hearing in the right ear and mixed hearing loss in the left ear with a 32-dB airbone gap and a $37 \mathrm{dBHL}$ air conduction threshold. Temporal bone CT findings showed a pneumatized mastoid cavity, although there was a soft tissue density which caused the eardrum to protrude outwardly, and filling of the inside of the mastoid antum, middle ear, and the epitympanum was observed. No structural damage of the ossicle, facial canal or lateral semicircular canal was identified (Fig. 2).

As left middle ear tumor or congenital cholesteatoma was suspected, we performed intact canal wall mastoidectomy 
with tympanization. According to the operative findings, a grayish-white multilobular mass and granulation tissue fully filling the mesotympanum, epitympanum, and mastoid cavity were detected (Fig. 3). The multilobular mass and granulation tissue were adjacent to the ossicle, although there was no ossicle erosion, and the hypotympanum and orifice of the Eustachian tube were preserved. No mass invasion into the sinus tympani was observed. Intact canal wall mastoidectomy was then performed, and the mass inside the epitympanium was excised by removing incus.

Tissue biopsy was performed to confirm the safety margin

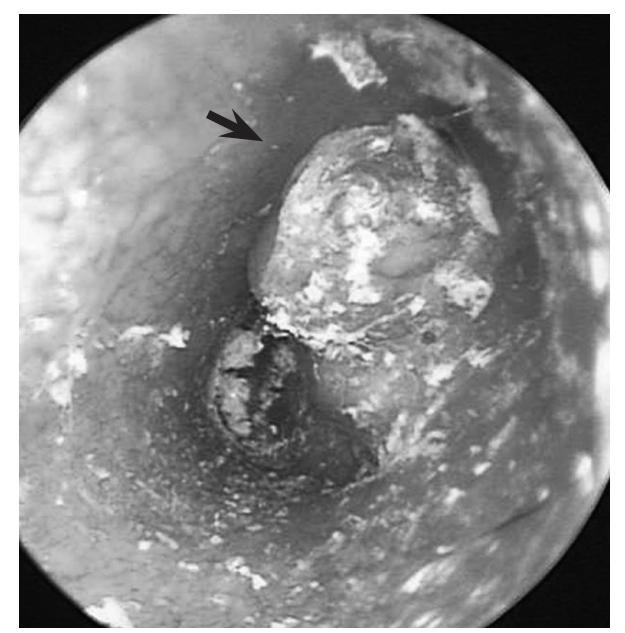

Fig. 1. The otoscopic finding of the patient's left ear indicates bulging of the posterosuperior portion of the ear drum (arrow) and external ear canal wall. at the outer surface of the tympanic membrane which protruded to the external auditory canal because of the mass, mucosa of hypotympanum and epitympanum.

In these tissues, no tumor cells were observed. Ossiculoplasty to restore hearing was not performed. Rather, a temporalis muscle facial graft was performed.

Postoperative histopathologic results revealed a small, round-cell tumor composed of even-sized, globular cells with plenty of eosinophilic cytoplasm arranged in a platy structure. There were no findings of mitosis, necrosis or dysplastic were.

To obtain the differentiation diagnosis of small, round-cell tumor, immunohistochemistry was performed. Based on the findings which were negative to the Ki-67 antigen, positive to synaptophysin, mildly positive to CD56, negative to chromogranin, and negative to S-100 protein, middle ear adenoma was diagnosed (Fig. 4).

In the three weeks following surgery, all of the packing material in the external auricular canal was removed, and two months after the surgery, the eardrum was well-grafted and stabilized. Two and seven months after surgery, temporal bone $\mathrm{CT}$ and pure tone audiometry were both performed. Pure-tone audiometry showed $8 \mathrm{dBHL}$ of the left bone conduction threshold and $32 \mathrm{~dB}$ of the air conduction threshold, both of which were similar to the patient's preoperative hearing level, however, the patient's tinnitus had improved. According to the temporal bone CT performed seven months after the surgery and otoscopy which was performed two years following the surgery, there was no evidence of recurrence.
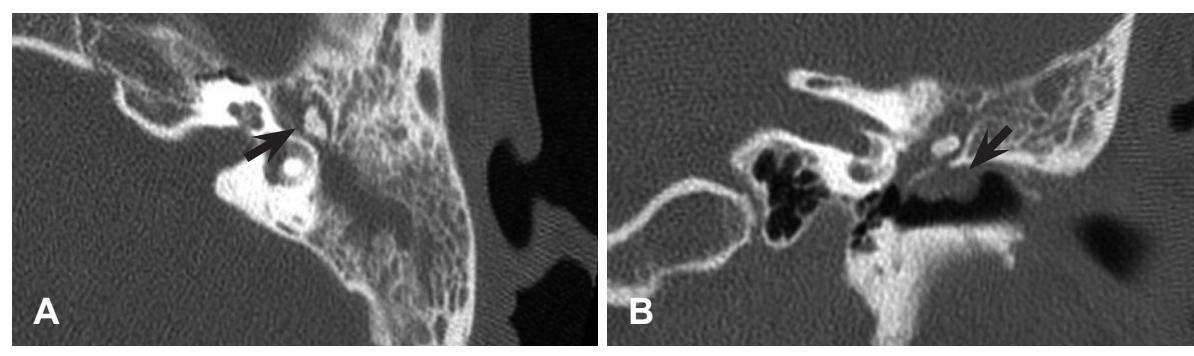

Fig. 2. Axial (A) and coronal (B) views of a computed tomography scan showing a soft-tissue mass in the left mastoid and middle ear. A: The ossicles are embedded in the mass (arrow). B: The posterosuperior portion of the tympanic membrane is displaced laterally by the mass (arrow).
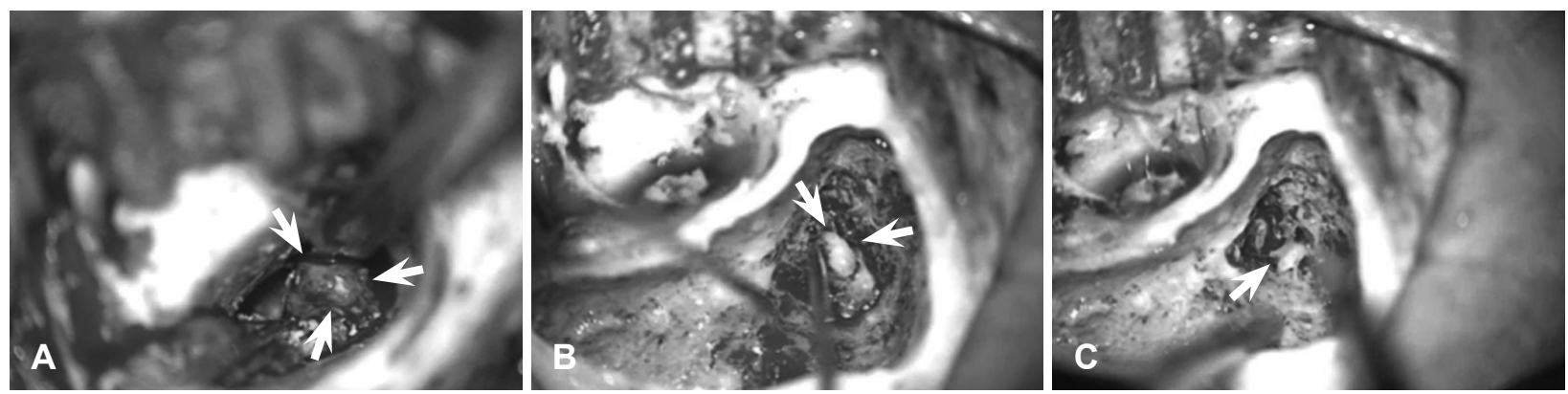

Fig. 3. Surgical finding reveals the tympanic cavity. A: The epitympanum and mesotympanum are filled with a white-grayish, polypoid mass (arrow) entraping the ossicles. B: The antrum is also filled by the mass (arrow). C: The mass in the epitympanum was removed together with the incus (arrow). 

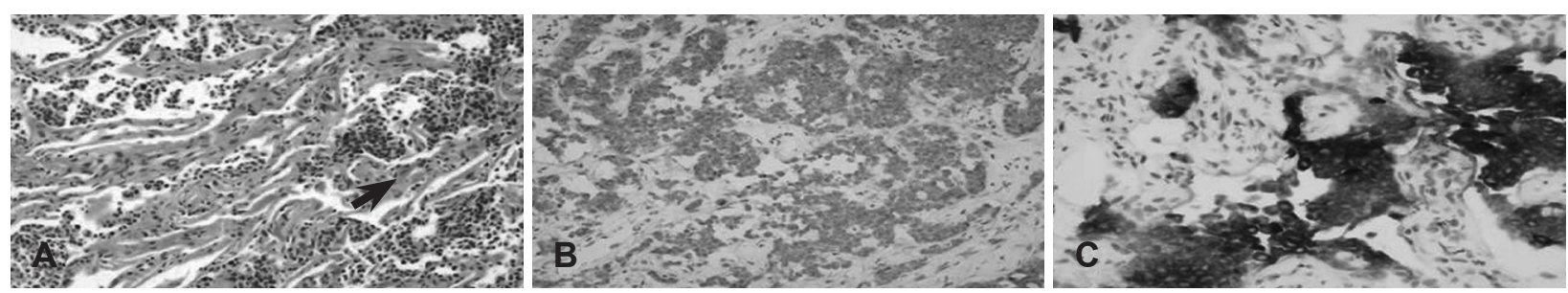

Fig. 4. Middle-ear adenoma. A: H\&E stain $(\times 200)$. B: CK7 immunohistochemistry $(\times 200)$. C: Synaptin immunohistochemistry $(\times 400)$. The tumor was composed of small, cuboidal cells containing eosinophilic cytoplasm. The stains for synaptophysin and CK7 were positive. The stains for chromogranin and S-100 proteins were negative. These findings support the diagnosis of middle-ear adenoma.

\section{Discussion}

Since benign tumors of middle ear glandular tissue were reported by Hyams and Michaels ${ }^{1)}$ in 1976, neuroendocrine tumor and middle ear adenoma have been separately described, but these tumors have been confused with ceruminoma, ceruminous adenocarcinoma, cystadenocarcinoma, ectopic salivary gland adenoma, adenocarcinoma, meningioma, and paraganglioma. Hypotheses such as the development of middle ear adenoma from the epithelial layer of the middle ear mucous gland $^{1)}$ and pluripotent endodermal stem cells located in the mucosa during the development of middle-ear-like pulmonary carcinoid tumor arising from Kulchitsky cells, ${ }^{7)}$ have been suggested. Another study reported the similarity between the developing process of middle ear carcinoid tumor and those of adenoma, although the details regarding the orifin of middle ear neuroendocrine cells are still unknown. However, opinions opposed to the idea of a similarity between middle ear carcinoid tumor and middle ear adenoma, have been also proposed. ${ }^{8)}$ And although it is still debatable, the opinions that middle ear adenoma can be differentiated from epithelial cells of mucous glands and neuroendocrine cells, ${ }^{9)}$ and that tumors can develop from pluripotent basal cells ${ }^{10)}$ or neural-crest-cellderived glomus tissue, ${ }^{1)}$ are widely supported these days. Accordingly, Saliba, et al. ${ }^{6)}$ classified types of adenomas of the middle ear as neuroendocrine adenoma, middle ear adenoma, and middle ear carcinoid tumor in accordance with the immunohistochemistry and the presence of metastasis to other organs which is a known clinical feature of middle ear carcinoid tumor. According to the cases which have been reported up to now, the mean onset age of middle ear adenoma is 44.6 years of age (16-77 years) with a male to female ratio of $1.3: 1$ in favor of males, and there was no difference between the right and the left ears. ${ }^{6}$ In previous Korean reports, the patient ages were 39 and 43, and which were older than in our patient. The most common patient complaint was unilateral hearing impairment, with majority of patients showing conductive hearing loss. In some cases, symptoms of aural fullness, ear pain, otorrhea, tinnitus, dizziness, and facial paralysis were report- ed. $^{3-5,7,12,13)}$

According to otoscopic findings, most of the cases showed a normal external auditory canal and eardrum. In the temporal bone CT findings, a soft tissue density surrounding ossicles and a relatively clear margin was observed. ${ }^{12)}$ MRI was performed for patients who were suspected of having a malignant tumor. The MRI findings showed higher signal of tumor than the white matter in the T1 sequence, the same signal as the gray matter in the $\mathrm{T} 2$ sequence, and an enhancing mass in the gadolinium-enhanced T1 sequence. ${ }^{14)}$

In our patient, a protruded mass was observed on otoscopy and a soft tissue density filling epitympanum and mastoid cavity were observed on CT, although ossicle erosion was not observed. Therefore, MRI was not performed based on the assumption that the tumor was benign.

The differential diagnosis of middle ear adenoma includes inflammatory diseases such as cholesteatoma and chronic otitis media, and primary middle ear tumor such as ceruminoma, pleomorphic adenoma, paraganglioma, jugular body tumor, and metastatic cancer. For the differential diagnosis, immunohistochemistry is usually performed.

A 6-90\% positive reaction to cytokeratin (such as CAM5.2, CK7 and CK20) which is an epithelial cell-derived probe, and a $15-88 \%$ positive reaction to chromogranin, neuron-specific enolase, synpatophysin, serotonin, human pancreatic polypeptide, and S-100 which is a neuroendocrine probe have been reported. ${ }^{15)}$ With the present case, as findings of positive to synaptophysin and CK7, negative to chromogranin and S-100, and partially positive to cytokeratin and neuroendocrine probe were reported, the patient was diagnosed as having middle ear adenoma accompanying neuroendocrine differentiation.

In most cases, surgical treatments are performed using a transmastoid approach with tympanoplasty. In some cases, 45-60 Gy of post-operative radiation therapy is performed, although chemotherapy is not performed. ${ }^{6,12)}$

In previous reports, the recurrence rate was low but the time for developing recurrence ranged widely from 12 months to 43 years. In all recurrence cases the initial treatments were focused on the procedures used to preserve the ossicle surround- 
ed by tumor and to remove the tumor only. ${ }^{6)}$

It is still controversial which is the most desirable treatment, although radical surgery such as complete removal of the tumor and ossicle, is considered to be able to reduce the recurrence rate. Considering the risk of developing a secondary cancerous tumor, the use of radiation therapy should also be given further consideration. ${ }^{6}$ The present case has been under observation for two years, during which no recurrence has been observed. However, considering the wide variety of recurrence times which have been reported in other studies, a long-term progress observation through routine physical exams and CT, is required.

\section{REFERENCES}

1) Hyams VJ, Michaels L. Benign adenomatous neoplasm (adenoma) of the middle ear. Clin Otolaryngol Allied Sci 1976;1:17-26.

2) Murphy GF, Pilch BZ, Dickersin GR, Goodman ML, Nadol JB Jr. Carcinoid tumor of the middle ear. Am J Clin Pathol 1980;73:816-23.

3) Jun KM. A case of primary adenocarcinoma of right middle ear cavity. Korean J Otolaryngol 1978;21:69-72.

4) Kwon J, Koh JW, Park SI, Jeong SH, Kim KY, Paik SI. A case of adenomatous tumor of the middle ear. Korean J Otolaryngol-Head Neck Surg 1991;34:1322-7.

5) Jun BH, Han YH, Yoon SP, Park SY. A case of the middle ear adeno- ma. Korean J Otolaryngol 2000;43:95-8.

6) Saliba I, Evrard AS. Middle ear glandular neoplasms: adenoma, carcinoma or adenoma with neuroendocrine differentiation: a case series. Cases J 2009;2:6508.

7) Batsakis JG. Adenomatous tumors of the middle ear. Ann Otol Rhinol Laryngol 1989;98:749-52.

8) Ramsey MJ, Nadol JB Jr, Pilch BZ, McKenna MJ. Carcinoid tumor of the middle ear: clinical features, recurrences, and metastases. Laryngoscope 2005;115:1660-6.

9) McNutt MA, Bolen JW. Adenomatous tumor of the middle ear. An ultrastructural and immunocytochemical study. Am J Clin Pathol 1985; 84:541-7.

10) Wassef M, Kanavaros P, Polivka M, Nemeth J, Monteil JP, Frachet B, et al. Middle ear adenoma. A tumor displaying mucinous and neuroendocrine differentiation. Am J Surg Pathol 1989;13:838-47.

11) Amble FR, Harner SG, Weiland LH, McDonald TJ, Facer GW. Middle ear adenoma and adenocarcinoma. Otolaryngol Head Neck Surg 1993;109:871-6.

12) Angouridakis N, Hytiroglou P, Markou K, Bouzakis A, Vital V. Middle ear adenoma/carcinoid tumour: a case report and review of the literature. Rev Laryngol Otol Rhinol (Bord) 2009;130:199-202.

13) Tabuchi K, Aoyagi $Y$, Uemaetomari I, Tobita T, Wada T, Inadome $Y$, et al. Carcinoid tumours of the middle ear. J Otolaryngol Head Neck Surg 2009;38:E91-4.

14) Maintz D, Stupp C, Krueger K, Wustrow J, Lackner K. MRI and CT of adenomatous tumours of the middle ear. Neuroradiology 2001;43: 58-61.

15) Berns S, Pearl G. Middle ear adenoma. Arch Pathol Lab Med 2006; 130:1067-9. 